\title{
Conocimientos, actitudes, prácticas y percepción de riesgo de los trabajadores de hatos lecheros de la Colonia 07 Montes frente a las zoonosis específicas
}

\author{
* Cynthia Núñez 1 , Margarita Samudio (iD) 1 \\ ${ }^{1}$ Universidad I beroamericana del Paraguay (UNIBE). Asunción-Paraguay
}

Cómo referenciar este artículo/

How to reference this article:
Núñez C, Samudio M. Conocimientos, actitudes, prácticas y percepción de riesgo de los trabajadores de hatos lecheros de la Colonia 07 Montes frente a las zoonosis específicas. Mem. Inst. Investig. Cienc. Salud. 2021; 19(2): 59-68

\begin{abstract}
RE S U ME N
Los trabajadores de hatos lecheros están en constante riesgo de contraer enfermedades zoonóticas como la brucelosis, tuberculosis, leptospirosis y carbunco bacteridiano, por la exposición a agentes biológicos y el contacto directo con animales durante el desarrollo de sus labores. Se describe el nivel de conocimientos, actitudes, prácticas, percepción de riesgo y las características sociodemográficas de los trabajadores de hatos lecheros de la colonia 07 Montes del distrito de Curuguaty, frente a las zoonosis, en el último trimestre del 2019. Fue un estudio cuantitativo, observacional, descriptivo, de corte transversal en el cual se utilizó un cuestionario con 33 preguntas aplicado a 121 trabajadores, con el que se recogieron variables demográficas, conocimientos, actitudes, prácticas y percepción de riesgo. Los datos se analizaron a través del programa estadístico SPSS. Para el análisis univariado, se empleó la estadística descriptiva para representar los datos expresados en frecuencias, porcentajes y tablas. El $52,9 \%$ de los trabajadores tenía conocimiento sobre la brucelosis, tuberculosis y leptospirosis, el 74,4\% tenía actitudes positivas, el $66,9 \%$ implementaba prácticas positivas y el $52,9 \%$ percibía el riesgo. Menos del $75 \%$ de los trabajadores encuestados tenían conocimientos, actitudes, prácticas y percepciones de riesgo óptimos, lo que resulta preocupante considerando la exposición constante de los mismos a las enfermedades zoonóticas, es por ello que se deberían implementar estrategias educativas de carácter multisectorial concernientes a estas patologías y sus medidas preventivas a modo de mejorar esta situación.

Palabras clave: zoonosis, conocimientos, prácticas, actitudes, percepción de riesgo, hatos lecheros, Paraguay.

\section{Knowledge, attitudes, practices and perception of risk of dairy herd workers in Colonia 07 Montes regarding specific zoonoses} * Autor correspondiente: Cynthia Núñez. Universidad I beroamericana del Paraguay (UNIBE). Asunción-Paraguay 
observational study, descriptive, cross-sectional, in which a questionnaire with 33 questions applied to 121 workers was used, with which demographic variables, knowledge, attitudes, practices and risk perception were collected. The data were analyzed through the SPSS statistical program. For the univariate analysis, descriptive statistics were used to represent the data expressed in frequencies, percentages and tables. It was observed that $52.9 \%$ of the workers had knowledge about zoonotic diseases, $74.4 \%$ had positive attitudes, $66.9 \%$ implemented positive practices and $52.9 \%$ perceived the risk. Less than $75 \%$ of the workers surveyed had optimal knowledge, attitudes, practices and perceptions of risk, which is worrying considering their constant exposure to zoonotic diseases, that is why multisectoral educational strategies should be implemented concerning these pathologies and their preventive measures to improve this situation.

Keywords: zoonoses, knowledge, practices, attitudes, perception of risk, dairy herds, Paraguay.

\section{NTRODUCCIÓN}

Se estima que existen alrededor de 1.415 microorganismos patógenos para el hombre, de ellos, entre el $61-65 \%$ son de origen animal y agentes zoonóticos ${ }^{(1)}$. Enfermedades como la brucelosis, tuberculosis, carbunco bacteridiano, entre otros, constituyen un problema de salud pública mundial que también ocasiona daños desde el punto de vista económico y social. Las ocurrencias de estas enfermedades se ven favorecidas por las condiciones ambientales, los altos índices pluviométricos y la alta probabilidad de contacto de personas y animales con los agentes patógenos ${ }^{(2)}$.

Con la naturaleza emergente de los patógenos zoonóticos, es trascendental que sea considerado el papel actual y futuro que pueden desempeñar los animales domésticos como fuentes potenciales de nuevas enfermedades ${ }^{(3)}$, y en este sentido, los ungulados son el hospedador no humano más importante, tanto en términos del número de especies zoonóticas patógenas soportadas como entre las especies zoonóticas emergentes y reemergentes ${ }^{(4)}$.

Los grupos con mayor exposición al ganado y los productos del ganado tienen un mayor riesgo de contraer infecciones zoonóticas bovinas, estos grupos incluyen a los trabajadores de hatos lecheros y personas que consumen leche no pasteurizada $u$ otros productos lácteos.

En Paraguay, Mendoza et $\mathrm{Al}^{(5)}$ realizaron un estudio observacional descriptivo de corte transversal con muestreo estratificado por conglomerado en el departamento de Caaguazú en el año 2018, donde encuestaron a los trabajadores de ambos sexos de los 360 hatos lecheros seleccionados al azar para evaluar el conocimiento, las prácticas de prevención y de bioseguridad sobre brucelosis. En Argentina, en el año 2016, Koziol et al. ${ }^{(6)}$ estudiaron el conocimiento sobre zoonosis de 203 operarios tamberos principal cuenca lechera bovina localizada en la Provincia de Santa Fe. Con el objetivo de evaluar la percepción de CAP de los productores rurales registrados en la Agencia de Defensa Agrícola del estado de Pará (ADEPARA) en Brasil, relacionados con la brucelosis bovina en sus rebaños, Gómes et al. ${ }^{(7)}$ realizaron un estudio transversal a través de la aplicación de cuestionarios a los productores rurales de 186 propiedades.

Uno de los principales rubros productivos de la Colonia 07 Montes de la localidad de Curuguaty constituye el sector lácteo, si bien existen pequeñas fincas en las que la leche producida es destinada al autoconsumo siendo comercializado el excedente, la mayor parte de los trabajadores están organizados en asociaciones y comisiones de producción lechera con centros de acopio. En los últimos años, los productores han evolucionado en aspectos productivos y asociativos, sin embargo, no se han implementado acciones tendientes a mejorar las condiciones de seguridad e higiene, que puede deberse a la ausencia absoluta de datos básicos sobre la frecuencia de zoonosis a los que están expuestos los productores lecheros por la actividad que realizan.

Para que la salud y seguridad laboral cumplan su objetivo, los trabajadores deben conocer y percibir el riesgo como tal, con la actualización metodológica y científica de los conocimientos y un enfoque actitudinal para el logro de prácticas preventivas 
positivas, pueden ser disminuidas las frecuencias de enfermedades zoonóticas. Actualmente, se están haciendo muchos esfuerzos para promover la producción lechera en la Colonia 07 Montes, pero hay información limitada sobre las enfermedades zoonóticas, incluida la concienciación y las prácticas públicas.

Por lo anterior, el desarrollo del estudio estuvo orientado a describir y relacionar el nivel de conocimientos, actitudes, prácticas, percepción de riesgo y las características sociodemográficas de los trabajadores de hatos lecheros de la colonia 07 Montes del distrito de Curuguaty frente a la zoonosis, en el último trimestre del 2019, lo cual, permitirá establecer estrategias preventivas promocionales de mayor efectividad.

\section{MATERI ALES Y MÉTODOS}

Se realizó un estudio observacional descriptivo de corte transversal, entre octubre a diciembre del año 2019, en la Colonia 07 Montes del distrito de Curuguaty, departamento de Canindeyú. El muestreo fue no probabilístico por conveniencia.

Fuente de datos. La técnica consistió en una encuesta que fue completada por las investigadoras según las respuestas brindadas en entrevistas personales de121 trabajadores de hatos lecheros en los locales de reuniones, centros de acopio y en los establecimientos agropecuarios. Se utilizó un cuestionario semiestructurado, con 33 preguntas, dividido en cinco secciones, basado en cuestionarios previos de trabajos similares ${ }^{(5-8-9)}$

a) Características demográficas del entrevistado: correspondió a los datos sociodemográficos del paciente, contiene 5 preguntas relacionadas con sexo del trabajador, edad (lista abierta), estado civil, escolaridad y antigüedad laboral (lista abierta).

b) Conocimientos: compuesto por 6 preguntas referentes al conocimiento de los trabajadores sobre la existencia de las enfermedades zoonóticas, identificación de por lo menos una enfermedad zoonótica, causas, signos clínicos y síntomas y prevención. Se consideró como conocimiento adecuado cuando el sujeto respondió correctamente el $60 \%$ o más (de 4 a 6 puntos) preguntas del cuestionario, y conocimiento no adecuado cuando obtuvo menos del $60 \%$ de respuestas correctas. Esta escala está basada en un trabajo previo similar ${ }^{(10)}$ y considerando la escala de notas académicas.

c) Actitudes: compuesta por 8 preguntas referentes a las actitudes tomadas por los trabajadores frente a las enfermedades zoonóticas considerando el reconocimiento como un problema, confidencialidad, estigmatización, deseo de recibir mayor información, existencia de tratamiento, automedicación en caso de síntomas, visita al médico en caso de sospecha, realización de pruebas en caso de aparición de síntomas. Se consideró como actitud positiva cuando el sujeto respondió correctamente el $60 \%$ o más (de 6 a 8 puntos) preguntas del cuestionario y como actitud negativa cuando obtuvo menos del $60 \%$ de respuestas correctas Esta escala está basada en un trabajo previo similar ${ }^{(10)}$ y considerando la escala de notas académicas.

d) Prácticas: compuesta por 10 preguntas referentes a las prácticas realizadas por los trabajadores en el desarrollo de sus actividades laborales, como desinfección de instrumentales, utilización de desinfectantes, eliminación de los desechos del tambo, consumo de leche cruda, frecuencia de utilización de elementos de protección personal. Se consideró como práctica positiva cuando el sujeto en estudio respondió correctamente el $60 \%$ o más (de 8 a 10 puntos) preguntas del cuestionario y como práctica negativa cuando obtuvo menos del $60 \%$ de respuestas correctas. Esta escala está basada en un trabajo previo $\operatorname{similar}^{(10)}$ y considerando la escala de notas académicas ${ }^{(10)}$.

e) Percepción de riesgo: compuesto por 4 preguntas referente a la percepción de los trabajadores considerando gravedad, mortandad, riesgo inmediato y responsabilidad frente a de las enfermedades zoonóticas. Se consideró como percepción de riesgo alto cuando el sujeto en estudio respondió correctamente el $60 \%$ o más (de 3 a 4 puntos) preguntas del cuestionario y como percepción de riesgo bajo cuando obtuvo menos del $60 \%$ de respuestas correctas. 
Análisis de datos. Se elaboró una base de datos con el software informático Excel, y los cálculos se realizaron con el software informático SPSS versión 19 para Windows. Se utilizaron medidas estadísticas de tendencia central y dispersión para las variables cuantitativas, número absoluto, tasas y porcentaje para las variables cualitativas.

Cuestiones éticas. Se respetaron los principios bioéticos y la investigación se llevó a cabo de acuerdo a las normas de la declaración de Helsinki ${ }^{(10)}$ cuando involucra participación de seres humanos. Todos los puntos y el objetivo de la investigación fueron explicados en su lengua materna, se respetó el anonimato de los trabajadores, explicándole que la información proporcionada sería confidencial y la participación voluntaria, dándole la opción de negarse. El estudio no presentó riesgo alguno ya que no se utilizó ninguna intervención en las variables fisiológicas y psicológicas y sociales de las personas participantes, tampoco representó un costo para ellos. Los resultados del estudio serán socializados con los sujetos encuestados, los datos obtenidos serán publicados para beneficio de la comunidad científica.

\section{RESULTADOS}

En este estudio fueron encuestados 121 trabajadores de hatos lecheros de la Colonia 07 Montes de la ciudad de Curuguaty, de entre 25 y 60 años de edad, entre los meses de octubre a diciembre del año 2019.

\section{Características sociodemográficas}

La mayoría $(81,8 \%)$ de los encuestados era del sexo femenino, estado civil casado $(91,7 \%)$, un rango de edad entre 25 y 67 años con una media de edad de 41,7 (DE $11,5)$ años, escolaridad primaria $(64,5 \%)$, y una antigüedad laboral promedio de 10,3 (DE 6,6) años (Tabla 1).

Tabla 1: Características demográficas de los trabajadores de hatos lecheros de la Colonia 07 Montes, Curuguaty, $2020(n=121)$

\begin{tabular}{llrr}
\hline Variable & Categoría & $\mathbf{n}$ & \% \\
\hline \multirow{3}{*}{ Sexo } & Masculino & 22 & 18,2 \\
\multirow{3}{*}{ Estado civil } & Femenino & 99 & 81,8 \\
& Casado/unión libre & 111 & 91,7 \\
Escolaridad & Soltero / divorciado & 10 & 8,3 \\
Edad (años) & Primaria & 78 & 64,5 \\
& Secundaria/Superior & 43 & 35,5 \\
Antigüedad laboral (años) & $\geq 10$ & 66 & 54,5 \\
& $<40$ & 55 & 45,5 \\
\hline
\end{tabular}

\section{Conocimientos sobre enfermedades zoonóticas}

En relación a los conocimientos de los trabajadores sobre las enfermedades zoonóticas se pudo constatar que el $61,2 \%$ de los mismos reconocen que existen enfermedades que son transmitidas de los animales al humano, el mismo porcentaje identificó por lo menos una enfermedad zoonótica como la brucelosis, 65,3\% conoce quienes pueden contraer las enfermedades zoonóticas, $66,1 \%$ reconocieron las causas, aunque un porcentaje bajo $(28,1 \%)$ identificó los signos clínicos y síntomas como la fiebre, el 46,3 \% manifestó que se pueden prevenir las enfermedades zoonóticas ya sea con la pasteurización de la leche $(7,4 \%)$ o la utilización de elementos de protección personal durante el desarrollo de las actividades que impliquen el contacto con animales $(38,9 \%)$. Tabla 2 
Tabla 2: Conocimiento de los trabajadores de hatos lecheros de la Colonia 07 Montes sobre las enfermedades zoonóticas, Curuguaty, $2020(n=121)$

\begin{tabular}{lcc}
\hline Aspectos conocidos por los encuestados & $\mathbf{n}$ & $\%$ \\
\hline Existen enfermedades que son trasmitidas de los animales al humano & 74 & 61,2 \\
Conoce por lo menos una enfermedad zoonótica especifica de hatos lecheros & 74 & 61,2 \\
Identifica brucelosis & 54 & 73,0 \\
Identifica tuberculosis & 15 & 20,2 \\
Identifica leptospirosis & 5 & 6,8 \\
Las personas que están en contacto directo con los animales pueden contraer & 79 & 65,3 \\
enfermedades zoonóticas & 80 & 66,1 \\
Conoce las causas de las enfermedades zoonóticas & 34 & 28,1 \\
Conoce los signos y síntomas de por lo menos una enfermedad zoonótica en el & 16 & 47,1 \\
humano & 13 & 38,2 \\
Fiebre ondulante (brucelosis) & 5 & 14,7 \\
Pérdida de peso (tuberculosis) & 56 & 46,3 \\
\hline Sufusión conjuntival (leptospirosis) & \\
Conoce la forma de prevención
\end{tabular}

\section{Actitud referente a los riesgos de enfermedades zoonóticas}

La mayoría $(66,1 \%)$ de los trabajadores encuestados en la presente investigación consideraron que las enfermedades zoonóticas constituyen un problema importante para su comunidad, un gran porcentaje de los mismos $(68,6 \%)$ mencionó que si un miembro de la familia contrajera alguna enfermedad zoonótica como la brucelosis, tuberculosis, leptospirosis, entre otros, no querría que la información permanezca de manera confidencial, el 73,6 \% resalta que si una persona contrae alguna enfermedad zoonótica no se le estigmatizaría por ello, al 86,8\% le gustaría recibir más información sobre estas enfermedades, el $86,8 \%$ cree que son enfermedades que pueden tratarse. En caso de aparición de síntomas, el 46,3 \% de los encuestados mencionó que no debemos automedicarnos y el $98,3 \%$ afirmó que hay que acudir al médico, el $43 \%$ consideraría la posibilidad de realizarse algún análisis laboratorial para detectar la enfermedad zoonótica (Tabla 3).

Tabla 3: Actitud de los trabajadores de hatos lecheros de la Colonia 07 Montes referente a los riesgos de enfermedades zoonóticas, Curuguaty, $2020(n=121)$

\begin{tabular}{|c|c|c|}
\hline Características & $\mathbf{n}$ & $\%$ \\
\hline $\begin{array}{l}\text { Considera que las enfermedades zoonóticas constituyen un asunto o } \\
\text { un problema importante para su comunidad }\end{array}$ & 80 & 66,1 \\
\hline $\begin{array}{l}\text { Si un miembro de la familia contrajera alguna enfermedad zoonótica, } \\
\text { no querría que esa información permaneciera de manera confidencial o } \\
\text { secreta }\end{array}$ & 83 & 68,6 \\
\hline $\begin{array}{l}\text { Si una persona contrae alguna enfermedad zoonótica, no se le } \\
\text { estigmatizaría por ese motivo }\end{array}$ & 89 & 73,6 \\
\hline Desea recibir más información sobre las enfermedades zoonóticas & 84 & 69,4 \\
\hline Considera que existe tratamiento contra las enfermedades zoonóticas & 105 & 86,8 \\
\hline $\begin{array}{l}\text { Considera que no debemos automedicarnos en caso de aparición de } \\
\text { síntomas }\end{array}$ & 56 & 46,3 \\
\hline $\begin{array}{l}\text { Considera que, si una persona sospecha que está infectada por alguna } \\
\text { enfermedad zoonótica, debe acudir a un médico }\end{array}$ & 119 & 98,3 \\
\hline $\begin{array}{l}\text { En caso de fiebre alta, consideraría la posibilidad de hacerse la prueba } \\
\text { de brucelosis, tuberculosis, leptospirosis u otra enfermedad zoonótica }\end{array}$ & 52 & 43 \\
\hline
\end{tabular}

\section{Prácticas implementadas por los trabajadores}

De la totalidad de los trabajadores encuestados, el 99,2 \% desinfecta sus materiales de trabajo ya sea con agua hervida o alcohol, el $76 \%$ utiliza antisépticos como el alcohol luego del lavado de manos al chequear un animal, el 70,2\% quema o 
entierra los desechos del tambo, el 90,9 \% no consume leche cruda. En cuanto a la frecuencia de la utilización de elementos de protección personal, el 69,4\% siempre utiliza guantes al realizar actividades laborales, el 13,2 \% utiliza gorro, 26,4 \% overol, $14,9 \%$ mascarilla, $15,7 \%$ protección ocular y el $87,6 \%$ siempre utiliza botas (Tabla 4).

Tabla 4: Prácticas implementadas por los trabajadores de la Colonia 07 Montes referente a los riesgos de enfermedades zoonóticas, Curuguaty, $2020(n=121)$

\begin{tabular}{lrr}
\hline Características & n & \% \\
\hline $\begin{array}{l}\text { Desinfecta los materiales de trabajo o instrumentales luego de } \\
\text { realizar una actividad laboral }\end{array}$ & 120 & 99,2 \\
$\begin{array}{l}\text { Utiliza antisépticos luego de lavar las manos al chequear un } \\
\text { animal }\end{array}$ & 92 & 76 \\
$\begin{array}{l}\text { Entierran los desechos del tambo } \\
\text { No consume leche cruda }\end{array}$ & 85 & 70,2 \\
\hline Siempre utiliza los guantes en su trabajo & 84 & 90,9 \\
\hline Siempre utiliza el gorro en su trabajo & 16 & 13,2 \\
\hline Siempre utiliza el overol en su trabajo & 32 & 26,4 \\
\hline Siempre utiliza la mascarilla en su trabajo & 18 & 14,9 \\
\hline Siempre utiliza protección ocular en su trabajo & 19 & 15,7 \\
\hline Siempre utiliza botas en su trabajo & 106 & 87,6 \\
\hline
\end{tabular}

\section{Percepción de riesgo}

Al analizar la percepción de riesgo de los 121 trabajadores encuestados, se determinó que el 49,6 \% consideró que las enfermedades zoonóticas son muy graves, pudiendo ocasionar la muerte $(46,3 \%)$, el $55,4 \%$ afirmó que existe el riesgo de contraerla en los próximos 6 meses, y el 50,4 \% consideró que la mayor responsabilidad de adoptar medidas de prevención para evitar las enfermedades zoonóticas es personal (Tabla 5).

Tabla 5. Percepción de riesgo de los trabajadores de hatos lecheros de la Colonia 07 Montes referente a los riesgos de enfermedades zoonóticas, Curuguaty, $2020(n=121)$

\begin{tabular}{llc}
\hline Características & n & \% \\
\hline Considera que las enfermedades zoonóticas son muy graves & 66 & 52,1 \\
Considera que las enfermedades zoonóticas pueden ocasionar la muerte & 63 & 52,1 \\
$\begin{array}{l}\text { Cree que existe el riesgo de que contraiga alguna enfermedad zoonótica } \\
\text { en los próximos } 6 \text { meses }\end{array}$ & 71 & 58,7 \\
$\begin{array}{l}\text { Considera que la mayor responsabilidad de adopción medidas de } \\
\text { prevención para evitar las enfermedades zoonóticas es personal }\end{array}$ & 65 & 53,7 \\
\hline
\end{tabular}

\section{DISCUSIÓN}

De la totalidad de los aspectos evaluados en relación a los conocimientos de los trabajadores de hatos lecheros de la Colonia 07 Montes, un importante porcentaje de los mismos $(52,9 \%)$ contestó de forma correcta por lo menos cuatro de las seis preguntas del cuestionario, considerando el $60 \%$, basado en una encuesta CAP aplicada en un estudio previo ${ }^{(10)}$. Aun cuando la mayoría de los mismos identificaron una enfermedad zoonótica y conocían las causas y quienes podrían contraerlas, un bajo porcentaje contestó de manera correcta sobre los signos, síntomas, lo que indica que se debe insistir en la capacitación de los trabajadores en relación a las enfermedades y riesgos a los que están expuestos.

Una proporción superior de conocimiento sobre leptospirosis fue reportada por Burgos et. al. ${ }^{(11)}$ en el año 2019, donde el 63,2 \% de las 714 personas encuestadas vinculadas a la cadena de producción bovina en la provincia Manabí, República del 
Ecuador, conocían sobre esta enfermedad zoonótica. En la presente investigación el $73 \%$ de los encuestados conocía sobre la brucelosis, coincidiendo con lo hallado por Castro et. $\mathrm{Al}^{(12)}$ en el que la mayoría de las amas de casa $(n=58)$ del ámbito rural de Rafaela, Santa Fe (2000) tenían conocimientos correctos sobre brucelosis con valores de $71 \%$.

También coincide con lo reportado por Luciano ${ }^{(9)}$ en el año 2011, quien mencionó que de la totalidad de los productores asociados a COTAPA $(n=75)$ de la Provincia de Entre Ríos-Argentina, la gran mayoría tenían conocimiento de la existencia de enfermedades zoonóticas y pudieron mencionar al menos tres. Sin embargo, no coincide con los resultados de otros investigadores como Piva et. al. ${ }^{(13)}$ y Mendoza et. al. ${ }^{(5)}$ quienes hallaron mayor porcentaje de trabajadores que no conocían sobre las enfermedades zoonóticas, con valores de $64,3 \%$ y $64 \%$ respectivamente. Estas diferencias pueden deberse a las capacitaciones constantes recibidas por los trabajadores de hatos en relación al manejo de los productos y las enfermedades zoonóticas, a través de las comisiones y asociaciones de producción lechera de la Colonia 07 Montes.

Podría considerarse que existe una actitud positiva de los trabajadores de hatos lecheros de la presente investigación, ya que el 74,4 \% de los mismos contestó correctamente por lo menos cinco de las ocho interrogantes propuestas. Considerando que una gran cantidad de personas trabajan con el rubro de la lechería en la comunidad, y un gran porcentaje de los mismos están asociados a cooperativas locales, los trabajadores encuestados identifican a las enfermedades zoonóticas como un asunto importante en la comunidad, ya que los mismos son conscientes de que la actividad realizada puede implicar el contagio a través de los animales. Además, no consideran que existiría una estigmatización por ello y desean recibir más información acerca de las enfermedades zoonóticas, evidenciando así, actitudes positivas hacia los riesgos a los que están expuestos.

Aunque un importante porcentaje de los trabajadores mencionaron que deben acudir a un médico en caso de sospechas, llamativamente, un porcentaje bajo de ellos considerarían la posibilidad de realizarse las pruebas de enfermedades zoonóticas como la brucelosis, tuberculosis o leptospirosis en caso de fiebre alta. Analizando tal actitud e influenciándola de manera favorable a través de capacitaciones, se podría facilitar la adopción de conductas seguras, que a su vez se reflejarían en resultados muy positivos en la disminución de los riesgos ocupacionales de carácter biológico. Es importante que los trabajadores estén convencidos de la adopción de estas actitudes positivas durante la realización de las actividades y los beneficios de la prevención para su salud, a modo de que las mismas sean constantes y duraderas con el tiempo.

Los resultados coinciden con lo hallado en el año 2007 por Sanabria et. al ${ }^{(14)}$, que al estudiar los conocimientos, actitudes y prácticas de 91 trabajadores rurales de la localidad de Lima-Perú sobre carbunco, hallaron una actitud mayoritariamente correcta de los mismos. Actitudes positivas también fueron halladas por Gomes ${ }^{(7)}$ en el año 2016, quien al estudiar los conocimientos, actitudes y prácticas de productores rurales pertenecientes a la Regional de Capanema en Pará, Brasil, determinó que el $66,66 \%(124 / 186)$ de los encuestados contestaron de manera correcta las interrogantes, a pesar de ello, el autor resaltó la necesidad de cambiar algunas prácticas para la protección eficiente, sugiriendo el mejoramiento de la educación sanitaria a modo de disminuir el comportamiento de riesgo de la población rural.

Resultados similares también fueron mencionados por Kulkarki et. al. ${ }^{(15)}$, donde el $77 \%$ de los 139 trabajadores rurales de Andhra Pradesh (India) encuestados en el año 2018, demostraron actitudes positivas al ser evaluados en relación a los conocimientos, actitudes y prácticas sobre las enfermedades zoonóticas, y aunque los mismos demostraron una actitud positiva hacia el uso de medidas de protección y seguridad, solo el $32 \%$ de los encuestados mostraron prácticas positivas, ya que no es probable que tomen las precauciones adecuadas, como el uso de guantes, el uso de botas, el chequeo veterinario regular, el uso de mascarillas, etc.

La mayoría $(66,9 \%)$ de los trabajadores de hatos lecheros de la Colonia 07 Montes implementan prácticas positivas en el desarrollo de sus actividades laborales ya que ese porcentaje contestó de forma correcta por lo menos 6 de las 10 interrogantes 
facilitadas en la encuesta, sin embargo, se observa que un porcentaje bajo de los mismos utiliza la totalidad de los elementos de protección personal como el gorro, overol, mascarilla y protección ocular, ocasionado de esta manera una exposición a la acción de los microorganismos patógenos causantes de enfermedades zoonóticas.

Las prácticas positivas como la utilización de elementos de protección personal, desinfección de las manos y materiales de trabajo, y la eliminación correcta de los desechos del tambo, adquieren relevancia cuando se maneja de que con ellas se puede evitar la transmisión de enfermedades como la brucelosis que, aunque se menciona una prevalencia oficial del $2 \%^{(16)}$ al $4 \%$, las mismas no reflejan el número real de animales infectados, estimándose que sería de 5 a 10 veces más alta que la notificada ${ }^{(5)}$. En este sentido, Della Valleet et al. $^{(17)}$ sostienen que la decisión de utilizar EPP podría basarse en factores como las normas sociales, la experiencia personal, la capacitación y podría ser impulsada por los rasgos de personalidad de aceptación de riesgos del trabajador.

Las botas constituyeron los elementos de protección personal más utilizados por los trabajadores encuestados $(87,6 \%)$, sin embargo, existe una baja frecuencia en la utilización de otros elementos como los gorros, protectores oculares, mascarillas y overoles, lo que coincide con lo observado por Caldas et al. ${ }^{(18)}$ en el año 2017, quienes hallaron que 22 de los 27 trabajadores rurales encuestados de los municipios de Araruna y Campos Mourao-Brasil, utilizaban botas durante el desarrollo de sus actividades, aunque ninguno de ellos utilizaba la totalidad de los elementos de protección personal necesarios ya que según lo mencionado por la mayoría de los mismos, no eran confortables, es por ello que los autores concluyeron que los trabajadores podrían estar expuestos a los posibles animales contaminados con enfermedades zoonóticas.

También coincide con el estudio de Koziol, et al. ${ }^{\left({ }^{6}\right)}$ en el año 2016, quienes mencionaron que los tamberos $(n=203)$ de la Provincia de Santa Fé-Argentina, poseían un tipo de comportamiento falto de sentido de autoprotección, al hallar que el uso de protector ocular (máscaras protectoras) y ropa específica fueron empleados por un bajo porcentaje de los tamberos. Lo mismo fue descripto por Luciano ${ }^{(9)}$ en el año 2011, al concluir que el uso de elementos de protección personal por los trabajadores productores tamberos de la Provincia de Entre Ríos-Argentina, era insuficiente ya que sólo el 54,3 \% utilizaba guantes, el 29,6\%, máscara/antiparras y el $19,4 \%$ ropa específica, donde los mismos encuestados reconocieron que estas actividades las tendrían que realizar de otra forma, con los elementos de protección correspondientes.

Un porcentaje alto $(99,2 \%)$ de los trabajadores realiza la práctica de desinfección de los materiales de trabajo, ya sea con agua hervida o alcohol, evitando la mayoría de los mismos a ocasionar una contaminación biológica de la leche cruda y con ello, vehiculizar zoonosis como la brucelosis y tuberculosis, ya que los microorganismos causantes de estas enfermedades son seres vivos imperceptibles a simple vista y se hallan en todas partes, incluyendo superficies de recipientes y utensilios.

Al considerar una escala del $60 \%$, se pudo evidenciar que la mayoría $(52,9 \%)$ de los trabajadores de hatos lecheros encuestados perciben el riesgo, lo que se puede deber a la actitud positiva que manifiestan los trabajadores en relación al riesgo biológico por enfermedades zoonóticas. Con relación a este punto, Espluga ${ }^{(19)}$ sostiene que el riesgo es un concepto formado por varias dimensiones, a las que cada individuo da mayor o menor importancia, en función de sus esquemas mentales (actitudes) y del contexto laboral concreto.

Koziol et al., en el año 2016, hallaron resultados distintos al evaluar la percepción de los riesgos en 203 operarios tamberos de la Provincia de Santa $\mathrm{Fe}^{(6)}$, en que los autores determinaron que la mayoría de las actividades como el ordeño, manejo de partos, abortos, y animales muertos fueron catalogadas por más de un tercio de los trabajadores como de bajo riesgo.

Una percepción baja de riesgo de los productores frente a los factores relacionados a tareas del tambo fue descripta por Luciano( ${ }^{(9)}$ en el año 2011 en la provincia de Entre Ríos, ya que un porcentaje muy bajo de los trabajadores manifestaron actividades como el manejo de partos, abortos y la manipulación de animales muertos como 
altamente riesgosas, resultados distintos a la presente investigación. Distinto al resultado de este trabajo, Swai et al. ${ }^{(20)}$ hallaron una menor cantidad de ganaderos $(\mathrm{n}$ $=43$ ) que percibían las actividades del tambo como riesgosas en relación a los trabajadores de salud animal $(n=36)(P<0.05)$ de Arusha y Tanga (Tanzania) en el año 2001, por lo que los autores concluyeron que el nivel de percepción era baja en relación a éstos últimos.

Cabe resaltar que un porcentaje importante de los participantes del estudio no tenía conocimiento sobre la existencia de las enfermedades zoonóticas, así como sus causas, síntomas y formas de prevención; también se destaca que una proporción baja de los mismos utilizaba la totalidad de los elementos de protección personal. Todo lo anterior es sumamente relevante al considerar la exposición permanente de los trabajadores a los agentes patógenos en el desarrollo de sus actividades laborales, es por ello que se debe insistir en la divulgación de informaciones concernientes a estas patologías y las medidas preventivas, esta problemática debe ser abordada con un enfoque multisectorial que involucre centros de salud, SENACSA, instituciones de Educación Superior, organizaciones no gubernamentales y aquellos relacionados con la cadena lechera como las cooperativas.

\section{AGRADECIMIENTOS}

A los trabajadores de hatos lecheros de la Colonia 07 Montes por acceder a la participación en el presente estudio.

Conflicto de interés: Las autoras declaran no tener conflicto de intereses.

Financiamiento: Las autoras declaran que no recibe financiación externa.

Contribución de los autores: Todos los autores han contribuido de igual manera para la investigación y la redacción del presente trabajo.

\section{REFERENCI AS BI BLI OGRÁFI CAS}

1. Cobos Valdes D, De Valle Fernández YT, Mulet NL, Martínez Martínez W, Rojas LP, De La M, et al. Elementos generales para analizar sobre las zoonosis. Correo Científico Med [Internet]. 2014 [cited 2018 Nov 26]; 18(4):710- Disponible en: http://scielo.sld.cu/pdf/ccm/v18n4/ ccm11414.pdf

2. Cintra F, García P, Hernández S, Pérez S. La zoonosis como Ciencia y su Impacto Social. Redvet [Internet]. 2006; VII (9): 19. Disponible en: https://www.redalyc.org/pdf/636/6 3612675013.pdf

3. Vega Aragón R. Zoonosis emergentes y reemergentes y principios básicos de control de zoonosis. Rev Med Vet (Bogota) [Internet]. 2009; 1(17): 8597. Disponible en:

http://www.scielo.org.co/pdf/rmv/n17/ n17a08.pdf

4. Martin C, Pastoret P, Brochier B, Humblet M, Saegerman C. A survey of the transmission of infectious diseases / infections between wild and domestic ungulates in Europe. Vet Res [Internet]. 2011; 42(70): 1-16. Disponible en: https://veterinaryresearch.biomedc entral.com/articles/10.1186/12979716-42-70

5. Mendoza L, Gonzalez L, Echeverria P, Idoyaga $\mathrm{H}$, Medina M, Gimenez $\mathrm{E}$, et al.
Conocimiento, prácticas de prevención y bioseguridad sobre Brucelosis en trabajadores de hatos lecheros. Rev salud publica Parag [Internet]. 2018; 8(2): 15-20. Disponible en: http://docs.bvsalud.org/biblioref/20 19/02/980487/21-27.pdf

6. Koziol EE, Molineri Al, Vanasco NB, Tarabla; Conocimiento de zoonosis en operarios tamberos de la provincia de Santa Fe, Argentina. Vet [Internet]. 2016 [cited 2017 Nov 6]; 18(1). Disponible en:

http://www.redalyc.org/pdf/1791/1791 49887006.pdf

7. Gomes N. Avaliação da percepção do conhecimento, atitude e prática dos produtos rurais, relacionada à brucelose em propriedades rurais pertencentes a Regional de Capanema, Pará, Brasil [Internet]. Universidade Federal do Pará; $2016 . \quad$ Disponible en: https://sucupira.capes.gov.br/sucup ira/public/consultas/coleta/trabalhoConc lusao/viewTrabalhoConclusao.jsf?popup =true\&id trabalho $=4682089$

8. Villacé MB, López L, Amieva M, Belfiore S, Estario M, Acosta L. Conocimientos, percepción de riesgo y comportamientos en relación con las zoonosis en adultos de la ciudad de 
Córdoba. Rev Argent Salud Pública. 2018; 9(36): 28-34.

9. Luciano C. Riesgo de accidentes y zoonosis: percepción y actitudes en productores tamberos asociados a cooperativa Tamberos Paraná de la provincia de Entreríos [Internet]. Universidad Nacional del Litoral; 2011 [cited 2017 Nov 6]. Disponible en: bibliotecavirtual.unl.edu.ar: $8080 /$ te sis/bitstream/handle/11185/542/\%20Te sis\% 20Ceci-final. pdf? sequence $=1$

10. Pasco C, Puescas P, Diaz C. Análisis de los conocimientos, actitudes y practicas preventivas de la población peruana femenina sobre el VIH-SIDA según encuesta demográfica y de salud familiar (ENDES) 2013- 2017. Rev cuerpo méd [Internet]. 2020; 13(3): 228-37.

Disponible en: https://docs. bvsalud.org/biblioref/2 021/04/1177957/729-otro-1443-2-1020201215.pdf

11. Burgos Macías DI, Pérez Ruano $M$, Bulnes Goicochea CA, Vera Mejía RR, Fonseca Rodríguez $O$. Nivel de conocimiento de la leptospirosis bovina en la provincia Manabí, Ecuador. Rev Salud Anim [Internet]. 2019; 41(2): 19. Disponible en:

http://revistas.censa.edu.cu/index.php/ RSA/article/view/1024/1327

12. Castro A, Tarabla H, Quaino $\mathrm{O}$. Conocimiento sobre brucelosis $y$ tuberculosis y su relación con algunas características de las amas de casa del área rural de Rafaela, Santa Fe. In: XIII o Reunión Anual, AsocArg VetLab Diag. Merlo, San Luis, Argentina; 2001.

13. Piva Filho GL, Alves AJS e, Carvalho LG, Marinho M, Queiroz LH. Ocorrência da brucelose e tuberculose bovina e percepção de riscos no Mato Grosso do Sul, Brasil. Arq Inst Biol (Sao Paulo) [Internet]. 2018; 84(0): 1-5.

Disponible en:

http://www.scielo.br/pdf/pvb/v32n11/a 11v32n11.pdf

14. Sanabria $H$, Riboty $A$, Villafuerte $A$, Gonzáles M. Conocimientos, actitudes y prácticas sobre carbunco: Estudio piloto en una zona endémica de Supe, Lima. Rev Peru Med Exp Salud Pública [Internet]. 2007; 24(1): 93-8.

Disponible en: http://www.scielo.org.pe/pdf/rins/v24n 1/a13v24n1.pdf

15. Kulkarni V, Venkata R. A cross sectional study of knowledge, attitude and practices regarding zoonotic diseases among agricultural workers. Public Heal Rev [Internet]. 2018; 5(2):71-6. Disponible en:

https://publichealth.medresearch.in/ind ex.php/ijphr/article/view/85

16. Naranjo J. Propuesta para la reformulación del Programa de Brucelosis bovina del SENACSA Paraguay [Internet]. Asunción; 2017 [cited 2018 Nov 28]. Disponible en: http://repositorio.iica.int/bitstream/ 11324/3165/1/BVE17089170e.pdf

17. Della Valle C, Hoppin J, Hines C, Andreotti G, Alavanja M. Uso de equipos de protección personal y de personalidad que aceptan riesgos dentro del Estudio de Salud Agrícola. Agromedicina [Internet]. 2012; 17(3): 264-76. Disponible en:

https://www.ncbi.nlm.nih.gov/pmc/artic les/PMC3425362/

18. Oliveira CC de, Ulbricht L, Moro ARP. Avaliação da exposição dos trabalhadores da Pecuária Leiteira aos riscos ocupacionais. Rev Uniandrade [Internet]. 2017; 18(1):1-15. Disponible en:

https://www. researchgate.net/publicati on/328247534 AVALIACAO DA EXPOS ICAO DOS TRĀBALHADORES DA PECU ARIA LEITËIRA AOS RISCOS OCUPAC IONAIS

19. Espluga JL, Trenc. NTP 415: Actos inseguros en el trabajo: guía de intervención [Internet]. España: Ministerio del Trabajo y Asuntos Sociales España; 1994. p. 10. Disponible en:

http://www.insht.es/Inshtweb/Contenid os/Documentacion/FichasTecnicas/NTP/ Ficheros/401a500/ntp 415.pdf

20. Swai, Schoonman \& $\bar{D}$. Knowledge and Attitude Towards Zoonoses among Animal Health Workers and Livestock Keepers in Arusha and Tanga, Tanzani. Tanzan J Health Res [Internet]. 2010; 12(4): 8. Disponible en: https://www.ncbi.nlm.nih.gov/pubmed/ $\underline{24409636}$ 